\title{
Seminare 2004
}

\section{Patientengerechtes Verhalten in der Arztpraxis}

\section{Teilnehmende}

Das Seminar richtet sich an das Medizinische Praxispersonal sowie an Ärztinnen und Ärzte.

Inhalt

Der erste Eindruck einer Patientin bzw. eines Patienten, welchen sie bzw. er von der Praxis vermittelt bekommt, ist für den Praxiserfolg sehr wichtig. Den Seminarteilnehmern/-innen wird aufgezeigt, wie dem Patienten ein «Kundengefühl» vermittelt wird und wie Reklamationen erledigt werden. Es wird darauf eingegangen, welches die Möglichkeiten des Telefons, als akustische Visitenkarte, und des persönlichen Patientenkontakts sind.

\section{Kosten}

Für Mitglieder des Schweizerischen Verbandes Medizinischer Praxis-Assistentinnen Fr. 285.Für übrige Teilnehmer/innen (MPA ohne Verbandsmitgliedschaft, Ärztin/Arzt) Fr. 300.-

\section{Daten}

Nr. 17 Donnerstag, 28. Oktober 2004 Hotel Bern Bern 9.00-17.45 Uhr

\section{Anmeldung}

Sie können sich bei nachfolgend aufgeführter Adresse (FMH Consulting Services) oder via Internet unter www.fmhservices.ch einschreiben.

\section{Röntgen in der Arztpraxis}

In der Schweiz röntgen 6000 Ärztinnen und Ärzte in ihrer Praxis. Beim Entscheid Röntgen in der Praxis ja oder nein beeinflussen emotionale Gründe vielfach mehr als sachliche Grundlagen. Zudem verunsichern Medieninformationen über den neuen Ärztetarif TARMED, den Praxisstopp oder die Aufhebung des Kontrahierungszwangs.

Mit diesem Seminar verschaffen wir Ihnen die Grundlage für Ihre persönliche Entscheidungsfindung. Dabei stützen sich unsere Referenten auf mehrere Dutzend Jahre einschlägige Erfahrungen, auf eine 10jährige minutiös ge- führte Statistik einer Landarztpraxis und auf Auswertungen von 60000 Aufnahmen.

Eine Checkliste und ein Fragenkatalog versetzen die Seminarteilnehmer/innen in die Lage, bei Ersatz- oder Neuinvestitionen kompetent aufzutreten und Vergleiche folgerichtig zu analysieren. Das Seminar erlaubt ihnen eine objektive Zukunftsbeurteilung auch im Hinblick auf die vom BAG angekündigten Neuerungen für das Röntgen ab dem 1. Januar 2008 - was heute bei deren Entscheidungen schon berücksichtigt werden muss.

\section{Teilnehmende}

Das Seminar richtet sich an Ärztinnen und Ärzte mit bestehender Praxis und an solche, die vor einer Praxiseröffnung oder Praxisübernahme stehen.

\section{Themen}

Das Seminar vermittelt einerseits Kenntnisse über die gesamte bestehende Röntgenthematik, andererseits werden klare Trends für die Praxis der nächsten Generation mit digitalem Röntgen aufgezeigt. Als spezifische Themen werden behandelt: Röntgentarife nach TARMED, Rentabilität Röntgen in der Arztpraxis, bestehende und neue Vorschriften ab 1. Januar 2008 (kann mit einem Wechsel der Anlage verbunden sein; Achtung bei Praxisübernahmen), Einstelltechniken für Fortgeschrittene, Spezialaufnahmen, Fehler erkennen und beheben, Hilfsmittel richtig einsetzen, wöchentliche Konstanzprüfung. Zudem wird eine Checkliste vorgestellt und durchgearbeitet für die Evaluation und die Beschaffung einer neuen Anlage.

\section{Unterlagen}

Die Seminarteilnehmer/innen erhalten ein Skript mit Zahlen einer Arztpraxis mit Kostenberechnung, Amortisation und interessanten Leasingformen (ab welcher Auslastung arbeite ich kostendeckend oder mit Gewinn?). Zudem erhalten sie eine Checkliste für die Evaluation und die Beschaffung einer neuen Röntgenanlage sowie eine CD-ROM oder ein Fachbuch mit über 50 Modellaufnahmen.

\section{Kosten}

Fr. 300.- (inkl. sämtlicher Kursunterlagen und Verpflegungen). 


\section{Daten}

Nr. 19 Donnerstag, 14. Oktober 2004 AusbildungsCham 9.30-16.00 Uhr

zentrum

Galexis

\section{Anmeldung}

Das Seminar ist auf maximal 15 Personen beschränkt. Sie können sich bei nachfolgend aufgeführter Adresse (FMH Consulting Services) oder via Internet unter www.fmhservices.ch einschreiben.

\section{Anmeldung und Auskunft}

FMH Consulting Services, Simone Köpfli, Burghöhe 1, 6208 Oberkirch, Tel. 04192500 77, Fax 0419210586 oder via Internet unter www.fmhservices.ch.

\section{Annullierungsbedingungen}

Bei Abmeldungen oder Fernbleiben werden folgende Unkostenbeiträge erhoben:

- $30 \%$ der Seminargebühren ab 14 Tage vor Seminarbeginn;

- $\quad 100 \%$ der Seminargebühren ab 7 Tage vor Seminarbeginn oder bei Fernbleiben. 